\title{
Article \\ Two-Dimensional Interference Estimator with Parallel Structure for Holographic Data Storage Channel
}

\author{
Thien An Nguyen (1) and Jaejin Lee *(1)
}

check for updates

Citation: Nguyen, T.A.; Lee, J. Two-Dimensional Interference Estimator with Parallel Structure for Holographic Data Storage Channel. Appl. Sci. 2022, 12, 2112. https:// doi.org/10.3390/app12042112

Academic Editor: Andrés Márquez

Received: 20 December 2021

Accepted: 16 February 2022

Published: 17 February 2022

Publisher's Note: MDPI stays neutral with regard to jurisdictional claims in published maps and institutional affiliations.

Copyright: (c) 2022 by the authors. Licensee MDPI, Basel, Switzerland. This article is an open access article distributed under the terms and conditions of the Creative Commons Attribution (CC BY) license (https:// creativecommons.org/licenses/by/ $4.0 /)$.
Department of Information Communication Convergence Technology, Soongsil University, Seoul 06978, Korea; anthienng1995@soongsil.ac.kr

* Correspondence: zlee@ssu.ac.kr; Tel.: +82-2-820-0901

\begin{abstract}
Recently, holographic data storage (HDS) has attracted significant attention as the nextgeneration storage technology because of its large capacity and high-speed transfer capabilities. However, two-dimensional (2D) inter-symbol interference (ISI) is a critical problem encountered in HDS. Therefore, in this study, we propose a 2D interference estimator to prevent the 2D ISI. In addition, we propose a parallel structure to exploit horizontal interference (HI) and vertical interference (VI) effectively. After detecting the $\mathrm{HI}$ and VI, the signal is combined using an average function. The simulation results indicate that the proposed model can significantly improve the performance of HDS.
\end{abstract}

Keywords: holographic data storage (HDS); estimating interference; removing interference; detection

\section{Introduction}

Many storage technologies have been proposed to satisfy the increasing demand for cloud storage. Particularly, research and development of holographic data storage (HDS) is being focused on as the next-generation storage technology [1,2]. In HDS, the data are stored as two-dimensional (2D) data pages. Thereafter, in the read process of the HDS system, the 2D data pages are captured as an image with a charge-coupled device (CCD) sensor [1]. Thus, the read-back data are distorted by 2D inter-symbol interference (ISI), pixel blur, misalignment, and noise [3].

To reduce the noise and improve the signal-to-noise (SNR) ratio, Gu et al. investigated the optimization of apertures [4]. In addition, to reduce the 2D ISI, other optical methods have been introduced for phase modulation [5,6] and polarization [7]. In addition, modulation and error correcting codes have been proposed to solve the 2D ISI [8-11]. Specifically, in signal processing, the equalizer combined with the partial response maximum likelihood (PRML) reduces the 2D ISI significantly [3,12-14].

Generally, PRML supplies coefficient interference to the soft output Viterbi algorithm (SOVA) to detect the received signal [15]. However, SOVA is designed to handle onedimensional (1D) ISI. Therefore, to obtain an effective SOVA, the 2D ISI needs to be converted into 1D ISI. The 2D ISI includes the interference from the upper, lower, left, and right directions. Therefore, the interference from the upper and lower directions can be removed to create a 1D ISI from the horizontal direction. Further, the interference from the left and right directions can be removed to create a 1D ISI from the vertical direction. Therefore, in this study, we proposed an interference estimator for estimating and removing interference to create the 1D ISI. In addition, we used the general partial response (GPR) target, which is an advanced version of PRML [16]. Moreover, we utilized a parallel structure to exploit horizontal interference (HI) and vertical interference (VI). Thereafter, we combined the information from HI and VI using an average function. During experiments, our proposed model significantly improved the bit error rate (BER) performance of HDS.

The remainder of this paper is organized as follows: Section 2 explains the 2D equalizer and GPR target. Section 3 presents an interference estimator. The proposed model is 
presented in Section 4. Section 5 presents and discusses the results of the simulation. Finally, Section 6 concludes the study.

\section{2D Equalizer and GPR Target}

During the training process, we applied the model shown in Figure 1 to estimate the coefficients of the equalizer and the GPR target. First, the original data $u[k]$ are modulated into the 2D signal $a[j, k]$, which is stored in a HDS channel. The received signal $y[j, k]$ from the output of the HDS channel and the 2D signal $a[j, k]$ are collected in the buffers. Thereafter, these data are supplied to the minimum mean square error (MMSE) algorithm.

As shown in Figure 1, we use equalizer $\mathbf{F}$ and GPR target $\mathbf{G}$ with a size of $5 \times 5$ and $3 \times 3$, respectively.

$$
\begin{gathered}
\mathbf{F}=\left[\begin{array}{ccccc}
f_{-2,-2} & f_{-2,-1} & f_{-2,0} & f_{-2,1} & f_{-2,2} \\
f_{-1,-2} & f_{-1,-1} & f_{-1,0} & f_{-1,1} & f_{-1,2} \\
f_{0,-2} & f_{0,-1} & f_{0,0} & f_{0,1} & f_{0,2} \\
f_{1,-2} & f_{1,-1} & f_{1,0} & f_{1,1} & f_{1,2} \\
f_{2,-2} & f_{2,-1} & f_{2,0} & f_{2,1} & f_{2,2}
\end{array}\right], \\
\mathbf{G}=\left[\begin{array}{cccc}
g_{-1,-1} & g_{-1,0} & g_{-1,1} \\
g_{0,-1} & g_{0,0} & g_{0,1} \\
g_{1,-1} & g_{1,0} & g_{1,1}
\end{array}\right] .
\end{gathered}
$$

Signals $z[j, k]$ and $d[j, k]$ are presented according to the following expressions:

$$
\begin{aligned}
& z[j, k]=\sum_{m=-2}^{2} \sum_{n=-2}^{2} y[j-m, k-n] f_{m, n}, \\
& d[j, k]=\sum_{m=-1}^{1} \sum_{n=-1}^{1} a[j-m, k-n] g_{m, n} .
\end{aligned}
$$

We can expand Equations (3) and (4) as follows:

$$
\begin{aligned}
& z[j, k]=y[j+2, k+2] f_{-2,-2}+y[j+2, k+1] f_{-2,-1} \\
& \ldots+y[j-2, k-1] f_{2,1}+y[j-2, k-2] f_{2,2} \\
& d[j, k]=a[j+1, k+1] g_{-1,-1}+a[j+1, k] g_{-1,0} . \\
& \quad \ldots+a[j-1, k] g_{1,0}+a[j-1, k-1] g_{1,1}
\end{aligned}
$$

To make Equations (5) and (6) shorter, we assign the below vectors:

$$
\begin{gathered}
\mathbf{y}=\left[\begin{array}{lll}
y[j+2, k+2] & \ldots & y[j-2, k-2
\end{array}\right]^{T}, \\
\mathbf{a}=\left[\begin{array}{llll}
a[j+1, k+1] & \ldots & a[j-1, k-1
\end{array}\right]^{T}, \\
\mathbf{f}=\left[\begin{array}{llll}
f_{-2,-2} & f_{-2,-1} & \ldots & f_{2,2}
\end{array}\right]^{T}, \\
\mathbf{g}=\left[\begin{array}{llll}
g_{-1,-1} & g_{-1,-0} & \ldots & g_{1,1}
\end{array}\right]^{T} .
\end{gathered}
$$

Based on the definitions from Equations (7)-(10), we can rewrite Equations (5) and (6) as follows:

$$
\begin{aligned}
& z[j, k]=\mathbf{f}^{T} \mathbf{y}, \\
& d[j, k]=\mathbf{g}^{T} \mathbf{a} .
\end{aligned}
$$

We calculate the mean square error (MSE) according to following expression:

$$
M S E=E\left\{\left(\mathbf{f}^{T} \mathbf{y}-\mathbf{g}^{T} \mathbf{a}\right)^{2}\right\} .
$$


To avoid $\mathbf{f}=\mathbf{g}=0$ for an optimal value of MSE in Equations (13), we should impose the condition for (13). Therefore, the optimal problem is as follows:

$$
\begin{aligned}
& \underset{\mathbf{f}, \mathbf{g}}{\arg \min }(M S E) \\
& \text { s.t. } \mathbf{E}^{T} \mathbf{g}=c
\end{aligned},
$$

where

$$
\begin{gathered}
\mathbf{E}^{T}=\left[\begin{array}{lllllllll}
0 & 0 & 0 & 0 & 1 & 0 & 0 & 0 & 0
\end{array}\right], \\
c=1 .
\end{gathered}
$$

We used the Lagrange function (17) and achieved $\mathbf{f}$ and $\mathbf{g}$ as follows:

$$
J=\mathbf{f}^{T} \mathbf{R} \mathbf{f}-2 \mathbf{f}^{T} \mathbf{T g}+\mathbf{g}^{T} \mathbf{A g}-2 \lambda\left(\mathbf{E}^{T} \mathbf{g}-c\right),
$$

where $\lambda$ is a vector containing the Lagrange multipliers, $\mathbf{A}=\mathbf{E}\left\{\mathbf{a a}^{T}\right\}, \mathbf{R}=\mathbf{E}\left\{\mathbf{y} \mathbf{y}^{T}\right\}$, and $\mathbf{T}=\mathbf{E}\left\{\mathbf{y a}^{T}\right\}$. Firstly, we calculate the partial derivatives, as shown below.

$$
\begin{gathered}
\frac{\partial J}{\partial \mathbf{f}}=2 \mathbf{R} \mathbf{f}-2 \mathbf{T g} \\
\frac{\partial J}{\partial \mathbf{g}}=-2 \mathbf{T}^{T} \mathbf{f}+2 \mathbf{A g}-2 \mathbf{E} \lambda \\
\frac{\partial J}{\partial \lambda}=-2\left(\mathbf{E}^{T} \mathbf{g}-c\right)
\end{gathered}
$$

Solving the equations $\frac{\partial J}{\partial \mathrm{f}}=0, \frac{\partial I}{\partial \mathrm{g}}=0$, and $\frac{\partial J}{\partial \lambda}=0$, we achieve the answer of (14), as shown below.

$$
\begin{gathered}
\lambda=\left(\mathbf{E}^{T}\left(\mathbf{A}-\mathbf{T}^{T} \mathbf{R}^{-1} \mathbf{T}\right)^{-1} \mathbf{E}\right)^{-1} c \\
\mathbf{g}=\left(\mathbf{A}-\mathbf{T}^{T} \mathbf{R}^{-1} \mathbf{T}\right)^{-1} \mathbf{E} \lambda \\
\mathbf{f}=\mathbf{R}^{-1} \mathbf{T} \mathbf{g} .
\end{gathered}
$$

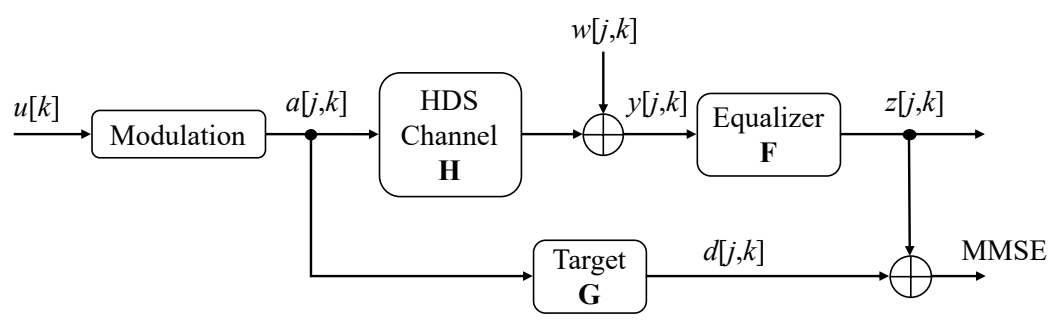

Figure 1. Model for the training process.

\section{Interference Estimator}

After the received signal $y[j, k]$ goes through the equalizer, it becomes approximately equal to signal $d[j, k]$. Thus, to design the $2 \mathrm{D}$ estimator, we can consider signal $d[j, k]$ in Equation (4) as follows:

$$
\begin{aligned}
& d[j, k]=\sum_{m=-1}^{1} \sum_{n=-1}^{1} a[j-m, k-n] g_{m, n} \\
& =a[j+1, k+1] g_{-1,-1}+a[j+1, k] g_{-1,0}+a[j+1, k-1] g_{-1,1} \\
& +a[j, k+1] g_{0,-1}+a[j, k] g_{0,0} \quad+a[j, k-1] g_{0,1} \\
& +a[j-1, k+1] g_{1,-1} \quad+a[j-1, k] g_{1,0}+a[j-1, k-1] g_{1,1}
\end{aligned}
$$


For the HDS channel, regularly, the estimated form of GPR target $\mathrm{G}$ is symmetric. Thus, we can assign matrix $\mathbf{G}$ as follows:

$$
\mathbf{G}=\left[\begin{array}{lll}
t & p & t \\
r & 1 & r \\
t & p & t
\end{array}\right],
$$

where $r=g_{0,-1}=g_{0,1}$ is the coefficient of interference from the horizontal direction; $p=g_{-1,0}=g_{1,0}$ is the coefficient of interference from the vertical direction; and $t=g_{-1,-1}=g_{-1,1}=g_{1,-1}=g_{1,1}$ is the coefficient of interference from the diagonal direction. We can rewrite Equation (24) as follows:

$$
\begin{aligned}
d[j, k] & =a[j+1, k+1] t+a[j+1, k] p+a[j+1, k-1] t \\
& +a[j, k+1] r+a[j, k]+a[j, k-1] r \\
& +a[j-1, k+1] t+a[j-1, k] p+a[j-1, k-1] t
\end{aligned}
$$

Based on Equation (26), we define left-right interference (LRI) as the interference from parameter $\left[\begin{array}{ccc}t & r & t\end{array}\right]^{T}$; upper-lower interference (ULI) as the interference from parameter $\left[\begin{array}{lll}t & p & t\end{array}\right] ; \mathrm{HI}$ as the interference from parameter $\left[\begin{array}{lll}r & 1 & r\end{array}\right]$; and $\mathrm{VI}$ as the interference from parameter $\left[\begin{array}{lll}p & 1 & p\end{array}\right]^{T}$. In addition, we make a group vector as follows.

$$
[v[j, k+1] \quad v[j, k] \quad v[j, k-1]],
$$

where

$$
\begin{gathered}
v[j, k+1]=a[j+1, k+1] t+a[j, k+1] r+a[j-1, k+1] t, \\
v[j, k]=a[j+1, k] p+a[j, k]+a[j-1, k] p, \\
v[j, k-1]=a[j+1, k-1] t+a[j, k-1] r+a[j-1, k-1] t .
\end{gathered}
$$

\begin{tabular}{|c|c|c|c|c|c|}
\hline $\begin{array}{l}a[j-1, k-1] / \\
a[j, k-1] / \\
a[j+1, k-1]\end{array}$ & $\begin{array}{l}a[j-1, k] / \\
a[j, k] / \\
a[j+1, k]\end{array}$ & $\begin{array}{l}a[j-1, k+1] / \\
a[j, k+1] / \\
a[j+1, k+1]\end{array}$ & $v[j, k-1]$ & $v[j, k]$ & $v[j, k+1]$ \\
\hline-1 & -1 & -1 & $-r-2 t$ & $-1-2 p$ & $-r-2 t$ \\
\hline-1 & -1 & 1 & \multirow{2}{*}{$-r$} & \multirow{2}{*}{-1} & \multirow{2}{*}{$-r$} \\
\hline 1 & -1 & -1 & & & \\
\hline-1 & 1 & -1 & $r-2 t$ & $1-2 p$ & $r-2 t$ \\
\hline 1 & -1 & 1 & $-r+2 t$ & $-1+2 p$ & $-r+2 t$ \\
\hline-1 & 1 & 1 & $r$ & 1 & $r$ \\
\hline 1 & 1 & -1 & & & \\
\hline 1 & 1 & 1 & $r+2 t$ & $1+2 p$ & $r+2 t$ \\
\hline
\end{tabular}

Because signal $a[j, k] \in\{-1,1\}$ and the interference is symmetric, $v[j, k+1], v[j, k]$ and $v[j, k-1]$ have six values, as listed Table 1.

Table 1. Results of $v[j, k]$ depending on $a[j, k]$.

From Table 1, we can design trellis to detect $v[j, k]$ using Viterbi algorithm (VA). The trellis has 36 states for $[v[j, k+1] v[j, k]]$, and each state has six output branches for $v[j, k-1]$. After determining the survivor path, we use $v[j, k+1]$ and $v[j, k-1]$ to achieve the LRI as follows:

$$
\operatorname{LRI}[j, k]=v[j, k+1]+v[j, k-1] .
$$


Additionally, we can make a group vector as follows:

$$
\left[\begin{array}{lll}
v[j+1, k] & v[j, k] & v[j-1, k]
\end{array}\right]^{T},
$$

where

$$
\begin{gathered}
v[j+1, k]=a[j+1, k+1] t+a[j+1, k] p+a[j+1, k-1] t, \\
v[j, k]=a[j, k+1] r+a[j, k]+a[j, k-1] r, \\
v[j, k-1]=a[j-1, k+1] t+a[j-1, k] p+a[j-1, k-1] t .
\end{gathered}
$$

Similarly, we can estimate ULI as follows:

$$
U L I[j, k]=v[j+1, k]+v[j-1, k]
$$

\section{Proposed Model}

The model proposed in this study is shown in Figure 2. The original data $u[k]$ are modulated into the data page $a[j, k]$, using a spatial light modulator (SLM), and stored in the HDS channel. The received data $y[j, k]$ is equalized into signal $z[j, k]$ using equalizer $\mathbf{F}$. Signal $z[j, k]$ is used to estimate LRI and ULI. Thereafter, we can remove the interference from signal $z[j, k]$ and apply SOVA to recover $\hat{a}[j, k]$. Finally, $\hat{a}[j, k]$ is demodulated to restore the original signal $\hat{u}[k]$. In Figure 2, the dashed line represents the signal during the training process. It did not work during the testing process.
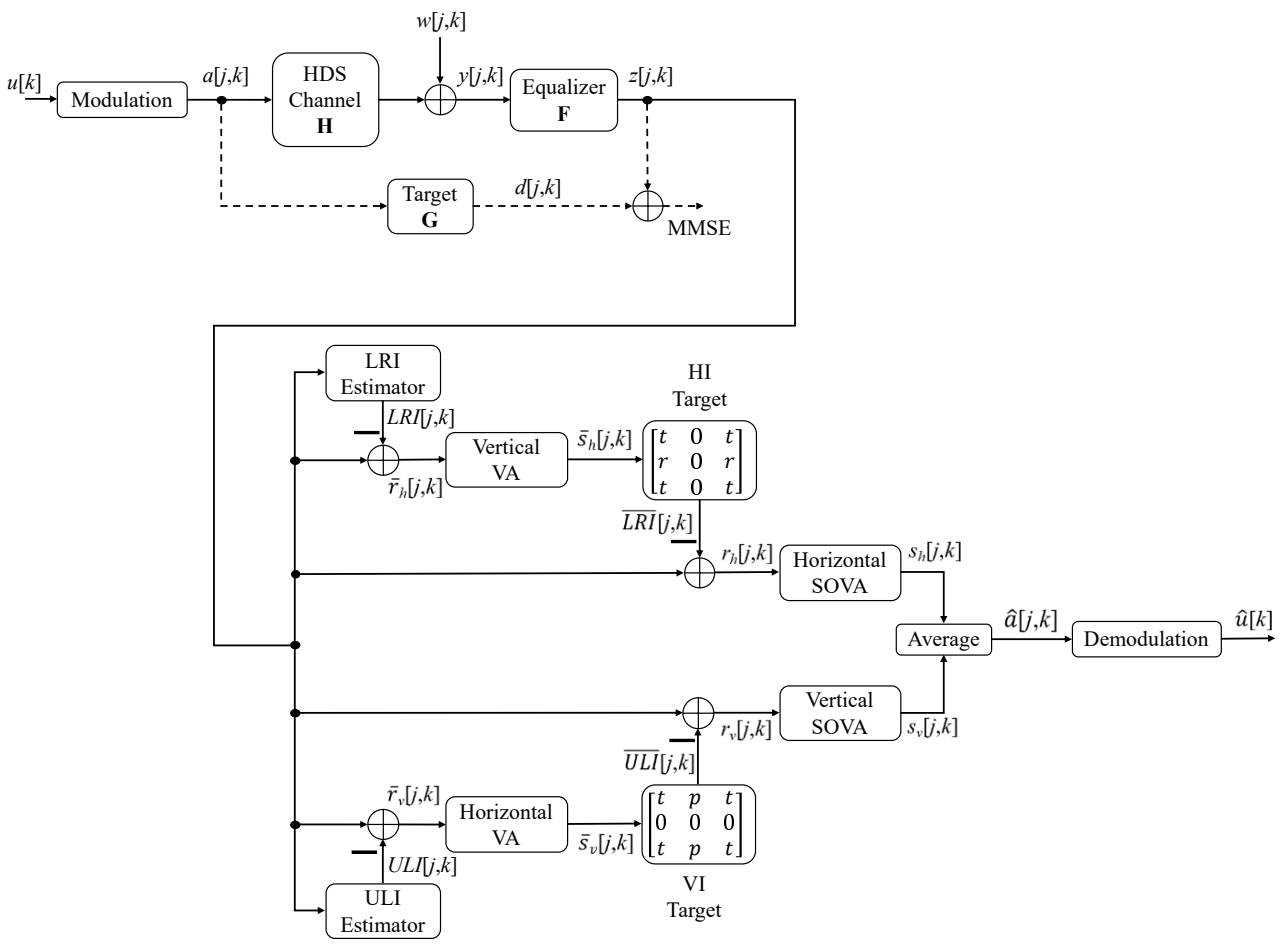

Figure 2. Proposed model.

\subsection{HDS Channel}

In this channel environment, the blurring effect and misalignment are modeled as a point spread function (PSF), and the noise is modeled as an additive white Gaussian noise (AWGN) $[3,17,18]$. 
The continuous PSF is presented as follows:

$$
h(x, y)=\frac{1}{\sigma_{b}^{2}} \sin c^{2}\left(\frac{x-m_{x}}{\sigma_{b}}, \frac{y-m_{y}}{\sigma_{b}}\right),
$$

where $\operatorname{sinc}(x, y)=(\sin (\pi x) / \pi x)(\sin (\pi y) / \pi y) ; \sigma_{b}$ is the blur grade; and $m_{x}$ and $m_{y}$ are the misalignments in the horizontal and vertical directions, respectively. The discrete PSF $h[j, k]$ can be calculated as follows:

$$
h[j, k]=\int_{j-\alpha / 2}^{j+\alpha / 2} \int_{k-\alpha / 2}^{k+\alpha / 2} h(x, y) d x d y
$$

where $\alpha$ denotes the linear fill factor. We investigate the influence of $\alpha$ in Section 5 . We consider that the range of the discrete PSF, $h[j, k]$, is $5 \times 5$ array pixels. The output of the HDS channel can be obtained as

$$
y[j, k]=a[j, k] \otimes h[j, k]+w[j, k]
$$

where $\otimes$ is the $2 \mathrm{D}$ convolution operator and $w[j, k]$ is the AWGN with zero mean and variance $\sigma_{w}^{2}$. Finally, we define (SNR) as follows:

$$
\mathrm{SNR}=10 \log _{10}\left(\frac{1}{\sigma_{w}^{2}}\right)
$$

\subsection{Detection}

We split the signal $z[j, k]$ into two branches; one is processed along the horizontal direction to extract the LRI, whereas the other is processed along the vertical direction to extract the ULI. Each branch includes an interference estimator, which removes the estimated interference and detects 1D interference. The interference estimator is presented in Section 3. The output of the interference estimator (i.e., LRI or ULI) is removed from the signal $z[j, k]$ to create signals $\bar{r}_{h}[j, k]$ and $\bar{r}_{v}[j, k]$, which are affected by the 1D interference (i.e., VI or HI). The estimated interference is removed according to the estimated signal $a[j, k]$. We consider Equation (31) as follows:

$$
\begin{aligned}
\operatorname{LRI}[j, k] & =v[j, k+1]+v[j, k-1] \\
= & a[j+1, k+1] t+a[j+1, k-1] t \\
+ & a[j, k+1] r+a[j, k-1] r \\
+ & a[j-1, k+1] t+a[j-1, k-1] t
\end{aligned}
$$

From Equation (41), we can use signal $a[j, k]$ to estimate the LRI. Therefore, we can apply VA to signal $\bar{r}_{h}[j, k]$ to achieve signal $\bar{s}_{h}[j, k]$, which is the estimated signal and is similar to signal $a[j, k]$. Thereafter, the signal $\bar{s}_{h}[j, k]$ goes through the VI target to achieve the estimated signal of the LRI (i.e., $\overline{L R I}[j, k]$ ) with higher accuracy. Similarly, we can improve the ULI and achieve signal $\overline{U L I}[j, k]$. We remove interferences $(\overline{L R I}[j, k]$ and $\overline{U L I}[j, k])$ from the signal $z[j, k]$ and apply SOVA to detect this signal. Finally, we use the average function to combine the output signals from the horizontal and vertical SOVAs. $\hat{u}[k]$ is obtained from the demodulated $\hat{a}[j, k]$.

\section{Simulation and Results}

Firstly, we randomly created the original data $u[k]$ with a size of $1,440,000$ bits and the same probabilities for 0 and 1 (OFF and ON). $u[k]$ is modulated into a data page of $1200 \times 1200$ bits. We used a spatial light modulator (SLM) to store the original data on the holographic medium. We used one page for the training process to estimate the equalizer and GPR target. During the testing process, ten pages were used to calculate the BER 
performance. The channel model of the HDS was characterized using an effective discrete PSF of size $5 \times 5$, as described in Section 4 .

We investigate the effect of the fill factor by varying $\alpha$ from 0.5 to 1 . During the first experiment, we set up an environment with a blur of 1.85 , and the $x$ - and $y$-axes misalignments were $0 \%$. The results are presented in Figure 3.

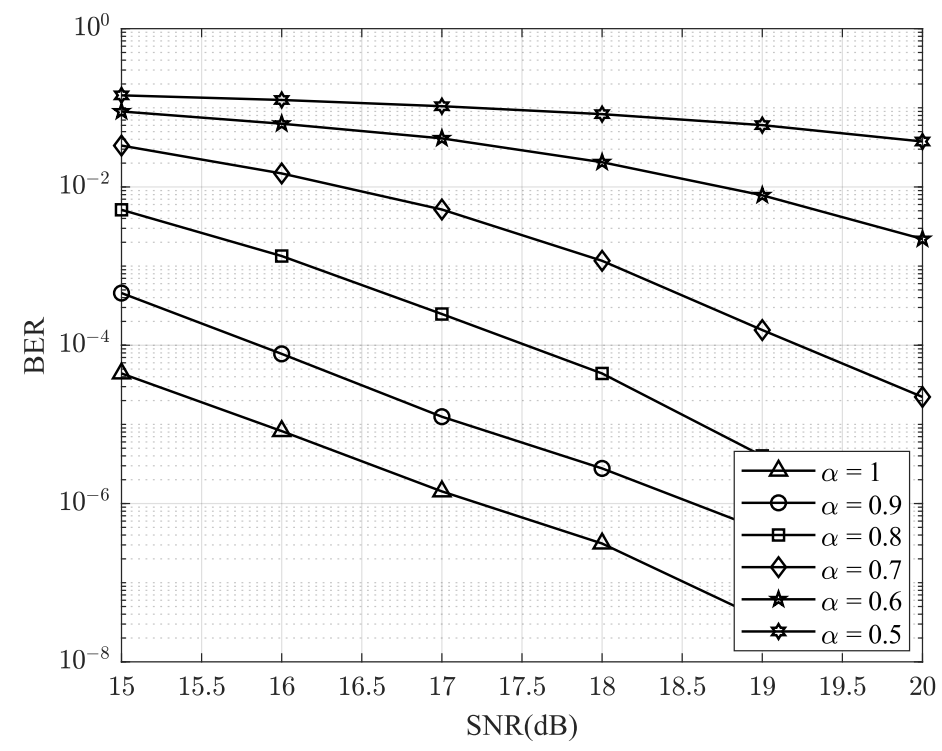

Figure 3. Effect of the fill factor on BER performance of the proposed model.

Figure 3 shows that the BER performance is worse as the fill factor $\alpha$ is smaller. When $\alpha$ decreases, the channel interference parameter also decreases because of the smaller intensity, and AWGN becomes the major factor in the received signal. In this article, for easier comparison, we chose $\alpha=1$ like the previous studies [17,18].

Next, we show the BER performance of the output signal from the horizontal SOVA, vertical SOVA, and average function (proposed model).

The results in Figure 4 indicate that the proposed model achieves the best performance when the horizontal process is combined with the vertical process using the average function. The gain is approximately $2 \mathrm{~dB}$ at a BER of $10^{-5}$.

In addition, we replace the linear equalizer with the nonlinear equalizers in $[19,20]$ to estimate the non-linearity in the channel. The results are shown in Figure 5.

Figure 5 shows that the BER performances of three equalizers are almost the same. This implies that the linearity is the major factor of the HDS channel during the simulation.

In the next experiment, the channel is affected by a blur grade of 2.0 and the a misalignment of 5\%. We compared our proposed model with a decision feedback equalizer [17] and a 2D SOVA [18]. The BER performance according to the SNR is depicted in Figure 6. 


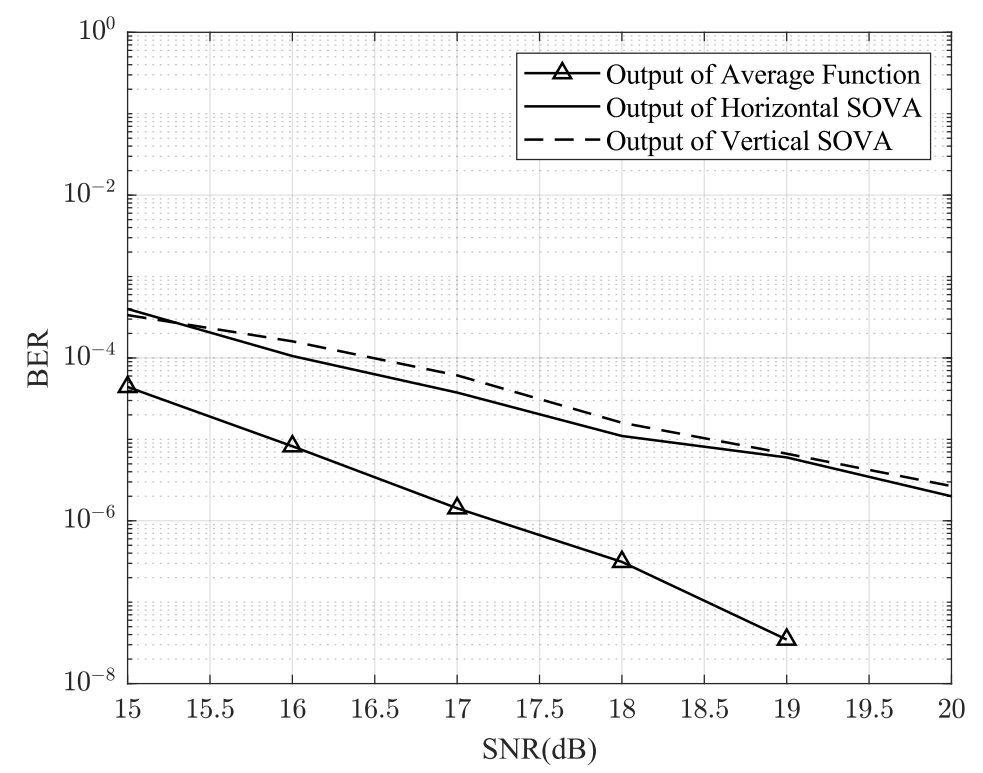

Figure 4. BER performance of the proposed model.

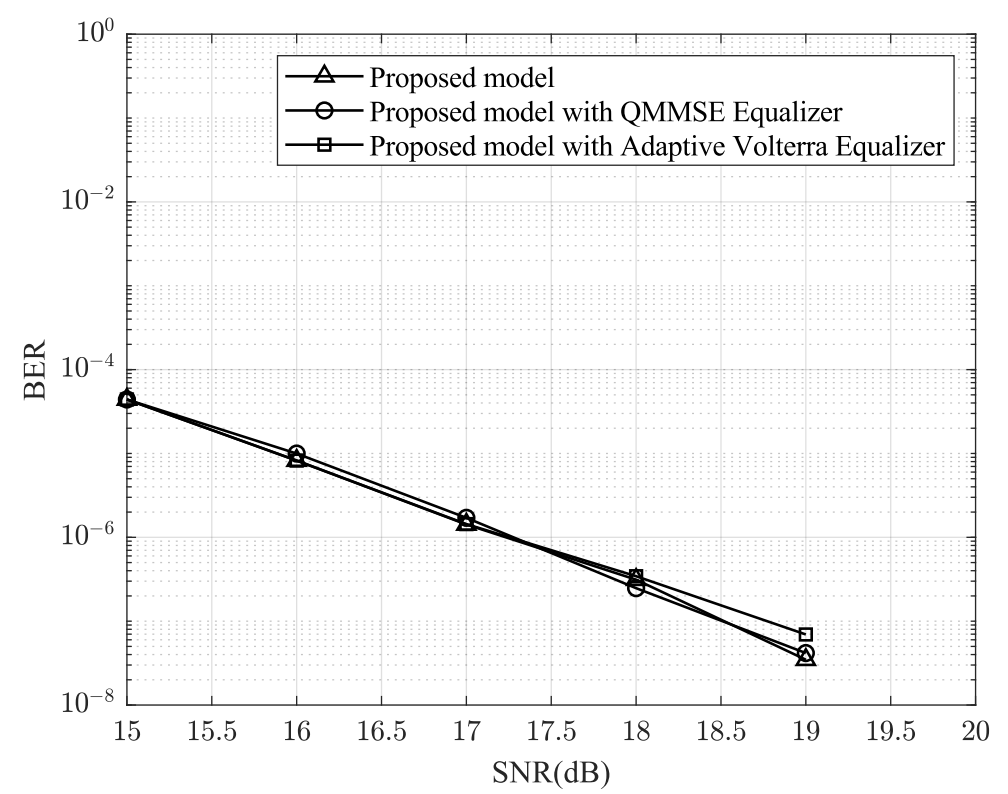

Figure 5. BER performance of the proposed model using the linear and nonlinear equalizers.

Figure 6 shows that our proposed model is better than that of previous studies. This is because our proposed model can estimate and remove the interference to make the SOVA effective. In addition, the average helps the proposed model utilize the information from horizontal and vertical processes.

Because blur is an important factor affecting HDS, we varied it from 1.9 to 2.1 with an SNR of $20 \mathrm{~dB}$ and a misalignment of $5 \%$. Figure 7 shows the BER performance according to the blur grade. 


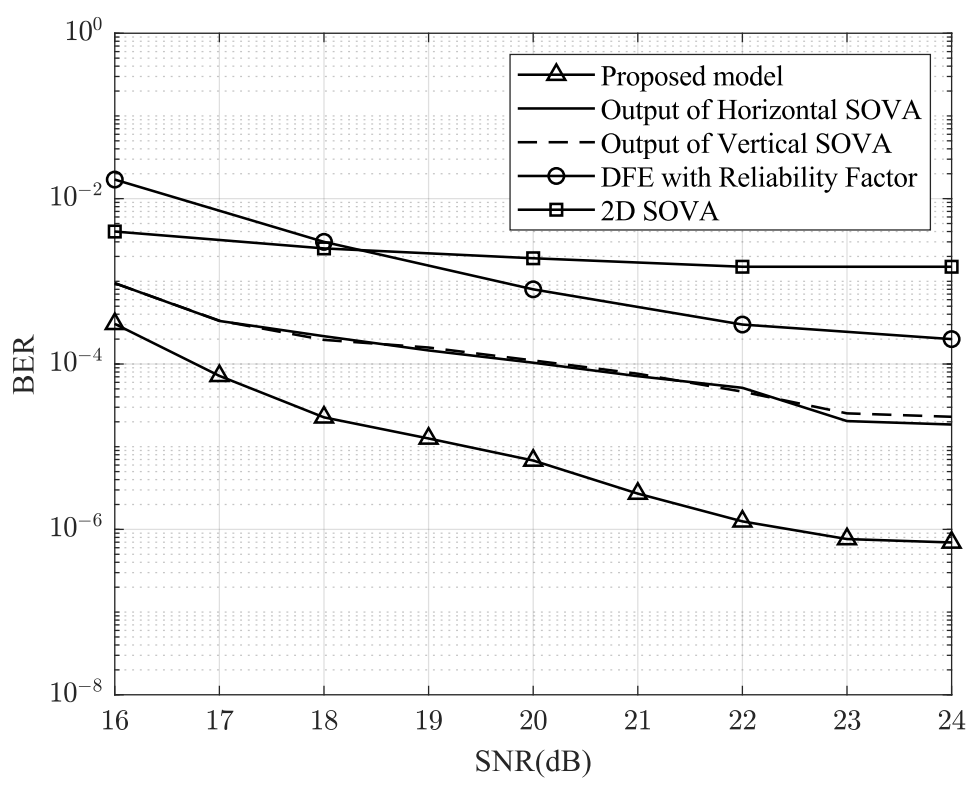

Figure 6. Comparison of the BER performances.

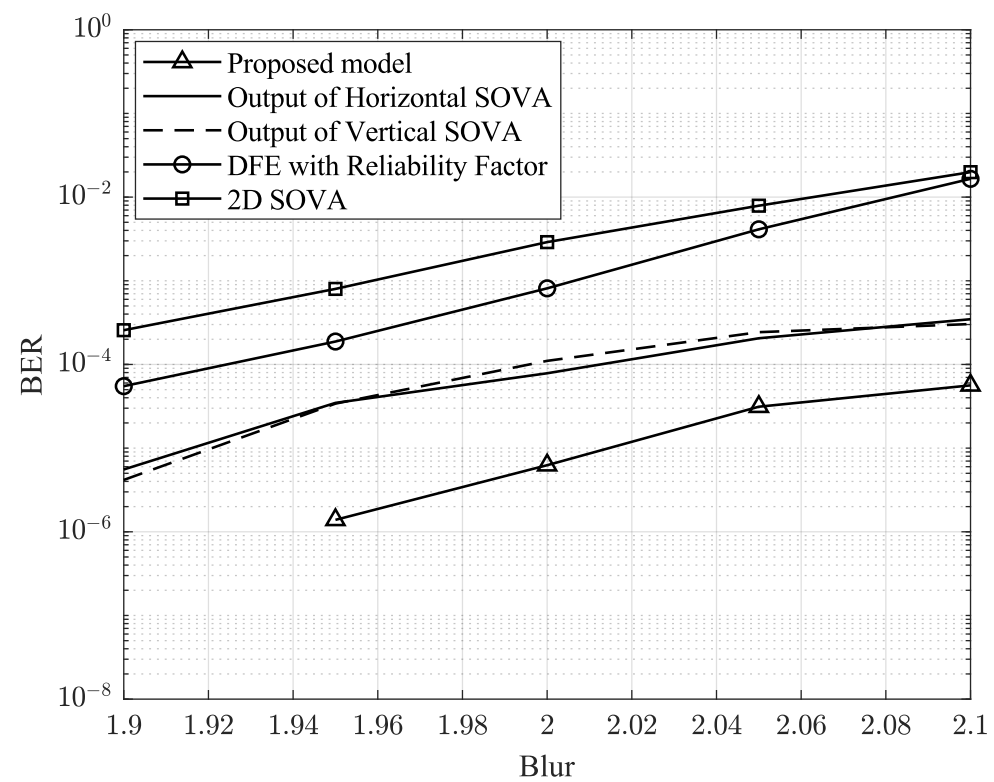

Figure 7. BER performance according to blur.

From Figure 7, we can observe that the BER performance of the proposed model is sensitive to blur. However, when compared with other methods, the proposed model achieves the best performance.

To investigate the effect of the $\mathrm{ON}$ and OFF ratio, we varied the probability of $\mathrm{ON}$ from 0.25 to 0.75 (or the probability of OFF from 0.75 to 0.25 ) at $15 \mathrm{~dB}$. The results are presented in Figure 8.

Figure 8 shows that the proposed model performs better when the probability of $\mathrm{ON}$ is smaller. 


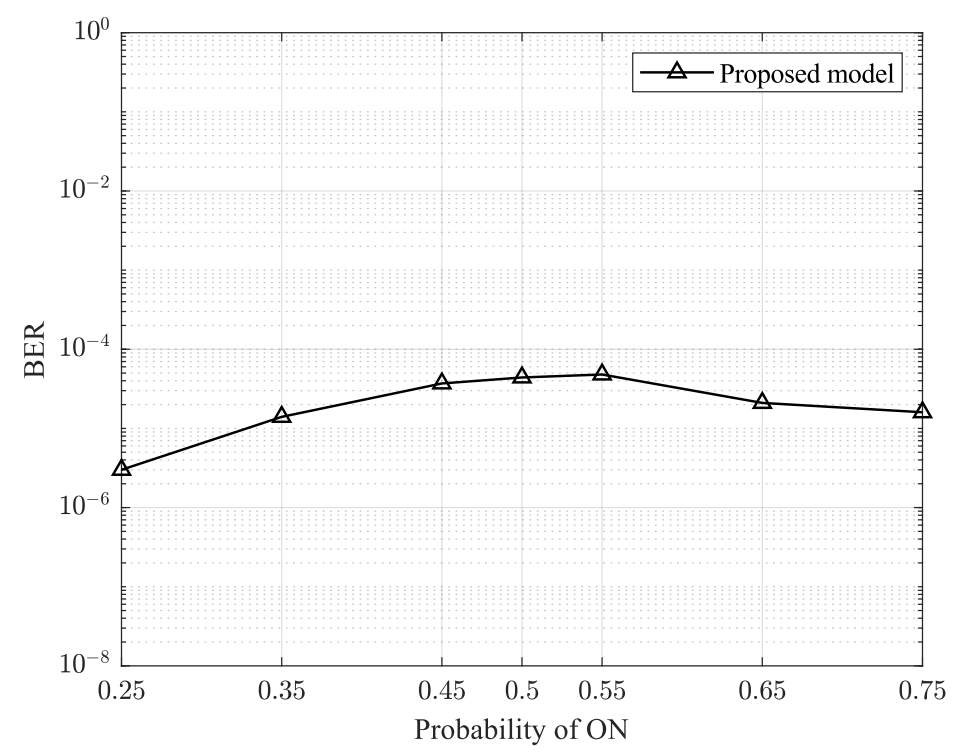

Figure 8. BER performance of the proposed model according to the $\mathrm{ON}$ and $\mathrm{OFF}$ ratio at $\mathrm{SNR}=15 \mathrm{~dB}$.

\section{Conclusions}

In this study, we proposed a 2D interference estimator. Firstly, we extracted the interference by the estimator using 2D VA. In this estimator, the 2D VA is used to detect the symbols, which are the sum of the product between the signal and the interference. Based on these symbols, LRI and ULI are estimated depending on the horizontal or vertical directions. With estimating LRI, we can remove it from the received signal and achieve the signal with the VI. By estimating ULI, we can achieve the signal with the HI. Therefore, we achieve the parallel structure, which includes the HI and VI. To improve the performance, we used the average function to combine the information from the horizontal and vertical processes (or HI and VI). The simulation results indicate that our proposed method can achieve a high gain compared to the models proposed in previous studies. Generally, in the proposed model, we exploit the 2D type target for extracting more accurate interference information between the equalizer output and the desired signal. Based on the target coefficients, we use interference estimator to convert 2D interference into 1D interference and utilize SOVA more effectively.

In the near future, we will replace the SOVA with the maximum a posteriori (MAP) algorithm. This makes the extrinsic information from two detectors be supplied to the prior information of the opposite MAP detectors.

Author Contributions: Conceptualization, T.A.N. and J.L.; methodology, T.A.N. and J.L.; software, T.A.N.; validation, T.A.N. and J.L.; formal analysis, T.A.N.; investigation, T.A.N. and J.L.; writingoriginal draft preparation, T.A.N.; writing—review and editing, T.A.N. and J.L.; supervision, J.L.; project administration, J.L.; funding acquisition, J.L. All authors have read and agreed to the published version of the manuscript.

Funding: This work was supported by the National Research Foundation of Korea(NRF) grant funded by the Korea government. (MSIT) (2021R1A2C1011154).

Institutional Review Board Statement: Not applicable

Informed Consent Statement: Not applicable

Data Availability Statement: Not applicable

Conflicts of Interest: The authors declare no conflict of interest. 


\section{References}

1. Hesselink, L.; Orlov, S.S.; Bashaw, M.C. Holographic data storage systems. Proc. IEEE 2004, 92, 1231-1280. [CrossRef]

2. Vadde, V.; Kumar, B.V.K.V. Channel modeling and estimation for intrapage equalization in pixel-matched volume holographic data storage. Appl. Opt. 1999, 38, 4374-4386. [CrossRef] [PubMed]

3. Koo, K.; Kim, S.V.; Jeong, J.J.; Kim, S.W. Two-Dimensional Soft Output Viterbi Algorithm with a Variable Reliability Factor for Holographic Data Storage. Jpn. J. Appl. Phys. 2013, 52, 09LE03. [CrossRef]

4. Gu, H.; Yin, S.; Tan. Q.; Cao, L.; He, Q.; Jin, G. Improving signal-to-noise ratio by use of a cross-shaped aperture in the holographic data storage system. Appl. Opt. 2009, 48, 6234-6240. [CrossRef]

5. Hara, M.; Tanaka, K.; Tokuyama, K.; Toishi, M.; Hirooka, K.; Fukumoto, A. Linear Reproduction of a Holographic Storage Channel using Coherent Addition of Optical DC Components. Jpn. J. Appl. Phys. 2008, 47, 5885. [CrossRef]

6. Ishii, T.; Fujimura, R. Interpixel crosstalk cancellation on holographic memory. Jpn. J. Appl. Phys. 2017, 56, 09NA10. [CrossRef]

7. Nobukawa, T.; Barada, D.; Nomura, T.; Fukuda, T. Orthogonal polarization encoding for reduction of interpixel cross talk in holographic data storage. Opt. Express 2017, 25, 22425-22439. [CrossRef] [PubMed]

8. Kim, J.; Lee, J. Two-Dimensional 5:8 Modulation Code for Holographic Data Storage. Jpn. J. Appl. Phys. 2009, 48, 03 A031. [CrossRef]

9. Kim, N.Y.; Lee, J.; Lee, J. Rate 5/9 Two-Dimensional Pseudo-balanced for Holographic Data Storage Systems. Jpn. J. Appl. Phys. 2006, 45, 1293. [CrossRef]

10. Chou, W.C.; Neifeld, M.A. Interleaving and error correction in volume holographic memory systems. Appl. Opt. 1998, 37, 6951-6968. [CrossRef] [PubMed]

11. Pishro-Nik, H.; Rahnavard, N.; Ha, J.; Fekri, F.; Adibi, A. Low-density parity-check codes for volume holographic memory system. Appl. Opt. 2003, 42, 861-870. [CrossRef] [PubMed]

12. Kim, J.; Lee, J. Partial Response Maximum Likelihood Detections Using Two-Dimensional Soft Output Viterbi Algorithm with Two-Dimensional Equalizer for Holographic Data Storage. Jpn. J. Appl. Phys. 2009, 48, 03A033. [CrossRef]

13. Koo, K.; Kim, S.-Y.; Kim, S.W. Modified two-dimensional soft output viterbi algorithm with two-dimensional partial response target for holographic data storage. Jpn. J. Appl. Phys. 2012, 51, 08JB03. [CrossRef]

14. Koo, K.; Kim, S.-Y.; Jeong, J.J.; Kim, S.W. Data page reconstruction method based on two-dimensional soft output Viterbi algorithm with self reference for holographic data storage. Opt. Rev. 2014, 21, 591-596. [CrossRef]

15. Hoeher, P.; Hagenauer, J. A Viterbi algorithm with soft-decision outputs and its applications. In Proceedings of the Global Telecommunications Conference and Exhibition 'Communications Technology for the 1990s and Beyond' (GLOBECOM), Dallas, TX, USA, 27-30 November 1989.

16. HNabavi, S.; Kumar, B.V.K.V. Two-Dimensional Generalized Partial Response Equalizer for Bit-Patterned Media. In Proceedings of the 2007 IEEE International Conference on Communications, Glasgow, UK, 24-28 June 2007.

17. Kim, K.; Kim, S.H.; Koo, G.; Seo, M.S.; Kim, S.W. Decision feedback equalizer for holographic data storage. Appl. Opt. 2018, 57, 4056-4066. [CrossRef] [PubMed]

18. Kim, J.; Lee, J. Two-Dimensional SOVA and LDPC Codes for Holographic Data Storage System. IEEE Trans. Magn. 2009, 45, 2260-2263.

19. He, A.; Mathew, G. Nonlinear equalization for holographic data storage systems. Appl. Opt. 2006, 45, 2731-2741. [CrossRef] [PubMed]

20. Nabavi, S.; Kumar, B.V.K.V. Application of linear and nonlinear equalization methods for holographic data storage. Jpn. J. Appl. Phys. 2006, 45, 1079-1083. [CrossRef] 